\title{
The Availability of Emergency Obstetric Care in Birthing Centres in Rural Nepal: A Cross-sectional Survey
} Amrit Banstola1 · Padam Simkhada2 · Edwin van Teijlingen3 · Surya Bhatta4 · Susma Lama5 • Abisha Adhikari4 •Ashik Banstola1,6

Please cite as:

Banstola, A., Simkhada, S., van Teijlingen, E., Bhatta, S., Lama, S., Adhikari, A., Banstola A., The availability of emergency obstetric care in birthing centres in rural Nepal: A crosssectional survey, Maternal \& Child Health Journal . http://link.springer.com/article/10.1007/s10995$\underline{019-02832-2}$

\section{Introduction}

Maternal and neonatal mortality are major public health problems worldwide as well as in Nepal. Despite substantial progress in reducing the Maternal Mortality Ratio (MMR) in Nepal from 539 to 239 maternal deaths per 100,000 between 1996 and 2016 (Ministry of Health et al. 2017; Pradhan et al. 1997), the MMR was the second highest in South-East Asia after Myanmar (WHO 2019). The neonatal mortality rate which was stagnant at 33 deaths per 1,000 live births for almost a decade (2001-2010) (Ministry of Health and Population et al. 2012, 2007) had declined to 21 deaths per 1,000 live births in 2016 (Ministry of Health et al. 2017).

The availability of Emergency Obstetric Care (EmOC) service is essential for increasing access to quality obstetric and neonatal care (Mkoka et al. 2014), increasing utilisation of maternal care services and institutional delivery (Rana et al. 2007), and ultimately reducing maternal and neonatal deaths (WHO et al. 2009). The availability of EmOC also indicates health system readiness to manage pregnancy and childbirth complications (Paxton et al. 2006; Travis et al. 2004). It has been estimated that with EmOC in place, up to 60\% of maternal deaths and $85 \%$ of intrapartum-related neonatal deaths could be averted per year (Lawn et al. 2009). 
The Government of Nepal is continuing to expand the availability and access to EmOC through public and private health facilities as mandated strongly by policies and strategies (Ministry of Health 2017, 2016b; Family Health Division 2006, 2002). The availability of EmOC demands a skilled birth attendant (SBA) and a provision of seven 'signal functions' for Basic EmOC (BEmOC): (1) administration of parenteral antibiotics; (2) administration of uterotonic drugs; (3) administration of parenteral anticonvulsants for pre-eclampsia and eclampsia; (4) manual removal of the placenta; (5) removal of retained products; (6) performing assisted vaginal delivery; and (7) basic neonatal resuscitation while Comprehensive EmOC (CEmOC) cover all seven BEmOC services plus (8) the ability to do a caesarean section; and (9) blood transfusion (WHO et al. 2009).

As such, health post (HP) and primary health care centre (PHCC) are strengthened to provide 24/7 delivery services and hospitals to provide CEmOC services in all 77 districts of Nepal. As a result, 2,101 (43\%) HPs and 188 (90\%) PHCCs were providing services regularly and a CEmOC site was established in 72 districts (but only 60 were functional) in 2018 (Ministry of Health and Population 2019). Nevertheless, nationally the percentage of EmOC met need was only 38\% in 2018 (Ministry of Health and Population 2019). The government's safe motherhood and neonatal health long-term plan (2006-2017) was to have delivery services in 70\% of HPs, BEmOC services in $80 \%$ of PHCCs, and CEmOC service in 60 districts by 2017 (Family Health Division 2006).

Very few studies have described the availability of human resources, medicines, and equipment to provide EmOC services in Nepal (Ministry of Health et al. 2017; Pradhan et al. 2010). Studies that have reported on the readiness of health facilities have focused on district- 


\section{Availability of emergency obstetric care}

level hospitals that provide CEmOC services only (Devkota et al. 2011) or limited to community-based birthing centres (without BEmOC and CEmOC) (Family Health Division 2014).

Currently, expansion and quality improvement of maternal and neonatal health service delivery at remote areas are being tested in Taplejung district (Ministry of Health 2016a). However, relatively little is known regarding the situation and readiness of health facilities to provide EmOC locally. Efforts to scale up maternal health services in this remote district and achieve the related Sustainable Development Goals (SDGs) have drawn attention to the need for evidence about service availability and readiness of birthing centres. Hence, our study aims to assess what birthing centres exist and how ready these are to provide EmOC services in Taplejung District.

\section{Methods}

\section{Study area and context}

The study was conducted in Taplejung; one of the remote mountainous districts in eastern Nepal. In 2016, the total number of women of reproductive age (15-49 years) was 37,965, the expected pregnancies and live births were 3,478 and 2,950 respectively (Ministry of Health 2016a). There were 62 health facilities (excluding private pharmacies and clinics) providing maternal and newborn care services at the time of the survey. Of these, 61 were public health facilities (one district hospital, two PHCCs, 50 HPs and eight community health units) and one was a private hospital.

According to the district health system, there is a provision of seven auxiliary nurse midwives (ANMs) (four non-SBA, three SBA), two operation theatre trained nurses, one anaesthesia 


\section{Availability of emergency obstetric care}

doctor/assistant, two obstetrics/gynaecologists, two medical officers, and nine paramedics in a district hospital that provides CEmOC services. Paramedics in Nepal includes health assistant (HA), auxiliary health worker (AHW), senior auxiliary health worker (Sr. AHW), laboratory technologist/officer/technician/assistant, radiographer, and darkroom assistant. Similarly, in PHCCs there were four paramedics (one HA, two AHWs/Sr.AHW, one laboratory technician/assistant), four ANMs (two non-SBAs, two SBAs) and one medical officer. In HP, which is a lower level of healthcare facility in Nepalese health system, there were two ANMs (at least one SBA in a health facility providing a delivery service) and three paramedics (one HA, two AHWs/Sr.AHW).

\section{Selection of the health facilities}

The survey involved all 16 public health facilities providing delivery services in the district (Supplementary Figure S1). This included one district hospital (15 bedded) designated to provide CEmOC, two PHCCs designated to provide BEmOC and 13 health posts designated to provide normal delivery services.

\section{Study design and data collection}

A cross-sectional health facility survey was conducted in 2018 using three data collection methods. First, data enumerators visited 16 birthing centres, and collected data using a structured survey tool. We used the core Service Availability and Readiness Assessment (SARA) questionnaire; the validated tool that has been designed to assess and monitor the service availability and readiness of health facilities. (WHO 2015).

Pre-testing of the survey questionnaire was done in two birthing centres of a neighbouring district. Following the pre-testing (van Teijlingen and Hundley 2005), adjustments were 


\section{Availability of emergency obstetric care}

made to the questionnaire to account for the information gained, resulting in the standard core questionnaire adapted for the district. Two enumerators and one field supervisor were mobilised to collect the data. The data collectors and field supervisor received one-day training before data collection. Data collectors obtained written informed consent in the local language before collecting the data from each concerned facility in-charge or nurse.

Secondly, data collectors observed the essential items that allowed us to determine the availability and the condition of equipment, medicines and commodities for EmOC. Finally, we extracted data from the Health Management Information Systems (HMIS) register of the included health facilities to determine the utilisation of EmOC signal functions and other maternal and newborn care services.

The ethical review board of Nepal Health Research Council approved this study (Reg. No. 435/2017) in December 2017.

\section{Data management and analysis}

The data collected on the paper questionnaire were checked for accuracy, completeness and consistency before entering electronically into Census and Survey Processing System (CSPro) Version 6.3 (WHO 2015). The complete data set was later analysed using SPSS version 24.

Descriptive statistics were used to assess the availability of EmOC services measured across domains (staff and guidelines, equipment, diagnostics (only in District Hospital), and medicines and commodities). The availability of EmOC services was also measured by determining the number of health facilities that performed the complete set of required signal 


\section{Availability of emergency obstetric care}

functions (seven for BEmOC and nine for CEmOC) in the three-month period before the assessment. Any facility providing at least one of the seven signal functions was considered as partially functioning BEmOC. Readiness scores were equal to the sum of the means that were obtained for each tracer item in a domain, divided by the total number of items in the domain, and then multiplied by 100. Readiness scores were calculated using unweighted averages.

\section{Results}

Availability of key health workers

Figure 1 shows the number of health workers at the time of assessment against the minimum staffing requirements for EmOC services. At the time of the survey, there were 117 health workers in 16 health facilities. Paramedics represented the largest category of staff ( $n=43 / 36.8 \%$ ). SBA trained nursing staff including auxiliary nurse midwives (ANMs) ( $n=33 / 28.2 \%)$ were the second largest cadre of health workers in the birthing centres. Six medical officers (5.1\%) were available in the District Hospital and PHCCs only. Obstetricians (2.6\%) and anaesthesia doctor/assistant (1.7\%) were available only in the District Hospital (Figure 1). Noticeably, the number of health workers in each group surpassed the number of sanctioned posts except for paramedics.

\section{Availability of EmOC signal functions}

Only the District Hospital offered CEmOC services (Table 1). None of the PHCCs could provide all seven signal functions. All 13 HPs had carried out at least one of the seven signal functions in three months before assessment (partially performing BEmOC). While the most commonly performed EmOC signal functions in three months before assessment by the surveyed health facilities was an administration of uterotonic drugs (87.5\%), the least 


\section{Availability of emergency obstetric care}

performed BEmOC signal function was an administration of parenteral anticonvulsants (12.5\%). None of the HPs studied used parenteral anticonvulsants. Similarly, none of the PHCCs expected to provide seven signal functions of BEmOC performed manual removal of placenta or assisted vaginal delivery during three months preceding the assessment.

\section{Reasons for not performing EmOC signal functions}

Except for the District Hospital, other surveyed health facilities did not perform a caesarean section and blood transfusion solely because of not fulfilling the policy requiring a specific infrastructure and trained providers to manage complicated deliveries (Figure 2). Although the reasons for not performing other seven signal functions were mixed, lack of case/patient requiring signal functions was predominant.

\section{Percentage of services utilisation in EmOC facilities}

Overall, 62.7\% ( $\mathrm{n}=662)$ of all institutional births occurred in CEmOC facility in the fiscal year 2016/17 (Table 2). The percentage of SBA delivery was also higher in CEmOC facility 59.4\% (538) compared to partially functioning BEmOC facilities 40.6\% (368). However, partially functioning BEmOC facility had the highest percentage of first ANC visits 60.5\% (617), fourth ANC visits 71.7\%\% (457) as per protocol (National Medical Standard for Reproductive Health Vol. II), pregnant women receiving Td2 vaccine 58.7\% (535), iron and folic acid 58.1\% (735) including women receiving de-worming tablets 56.8\% (698).

A total of 94 obstetric complications were recorded at all surveyed health facilities; HP (20.2\%), PHCCs (2.1\%) and district hospital (77.7\%). Postpartum haemorrhage (39.4\%), preeclampsia/eclampsia (14.9\%), puerperal sepsis (12.8\%) and prolonged labour (12.8\%) were the main obstetric complications (Table 2). 


\section{Availability of selected essential equipment}

Essential equipment for performing some EmOC functions was not available in all facilities (Table 3). Complete delivery packs, vacuum aspiration (MVA) kits, and blank partographs were equally available at $87.5 \%$ (14/16). Oxygen supply (18.8\%) was the least common equipment in the surveyed health facilities. Overall, the availability of equipment varied depending on the type of health facility. Equipment was more frequently available in the District Hospital and PHCCs than in HPs.

\section{Availability of selected essential medicines and commodities}

Chlorhexidine, oxytocin, magnesium sulphate and intravenous solution with infusion set (without dextrose) were equally available at $87.5 \%$ (14/16) of health facilities on the day of the survey (Table 4). Gentamicin injection 31.3\% (5/16) was least available in all health facilities offering EmOC included. While PHCCs lacked ampicillin injections, the district hospital was deficient in xylocaine, blood supply and other essential medicines (e.g., halothane, thiopental).

\section{Obstetric service readiness in health facilities}

The overall readiness score was $76.8 \%$ for providing BEmOC and the highest was for equipment (87.1\%) and the lowest for staff and guidelines (51.3\%) as shown in Figure 3. There was difference in the BEmOC readiness score by type of health facility. A higher-level health facility (District Hospital) had a higher readiness score than a lower-level health facility (HP), $95.8 \%$ versus $74.0 \%$. Figure 4 shows the overall readiness score to provide CEmOC was 70.0\% and highest for staff and guidelines (100.0\%) and diagnostics (100.0\%), and the lowest for medicines and commodities (44.4\%). 


\section{Geographic distribution of EmOC facilities}

The distribution of EmOC services varied across the district. Only one available CEmOC facility was mainly concentrated in Phungling Municipality (district headquarter). Partially performing BEmOC facilities were mainly available in rural municipalities of South East and South West regions as shown in Supplementary Figure S1. All these facilities were located in hilly areas with an elevation ranging between 1,295 to 2,484 meters.

\section{Discussion}

This study provides detailed information on the availability of staff, equipment, medicines and commodities along with readiness of birthing centres to provide EmOC services in Taplejung District. The District Hospital provided nine signal functions of CEmOC for 129,767 people at the time of the study. Other fifteen were found to be partially functioning BEmOC facilities, as they did not provide all the seven signal functions. The WHO handbook on monitoring EmOC recommends the minimum of five EmOC facilities with at least one CEmOC per 500,000 population (WHO et al. 2009). According to the standard set by the guideline, our study has shown that overall the minimum acceptable level of EmOC services in Taplejung has not been met, although, District Hospital offers CEmOC.

The District Hospital, as a consequence was overcrowded with deliveries. The study found that in the fiscal year 2016/17, 62.7\% of total institutional deliveries in the district took place in the District Hospital. This relatively higher percentage of women delivering in the District Hospital irrespective of a lower percentage of fourth ANC visits as per protocol (28.3\%) than in partially functioning BEmOC (71.7\%) has several explanations. First, it is important to note that this hospital handles most of the complicated cases referred from other health 


\section{Availability of emergency obstetric care}

facilities within the district. In addition, as other partially performing EmOC services were located mainly in the remote hilly areas, the pregnant women and their families due to remoteness and lack of comprehensive services including caesarean section may less prefer them (Anastasi et al. 2015; Bohren et al. 2014).

Most of the EmOC services in PHCCs are underutilised which could be linked to the lack of necessary facilities such as operating theatre to handle complicated cases despite the availability of a doctor. The low availability and readiness of EmOC services in HPs may encourage women to deliver at home without the assistance of SBA (Roro et al. 2014). There may also be an increased risk of bypassing local birth centres and delivering at urban health facilities (Karkee et al. 2015).

Unlike the studies conducted in Nepal and other LMICs (Low and Middle Income Countries), our study found that Taplejung District has good availability of key health personnel for providing EmOC services. It might be due to the determination of the leadership of District Health Office to fulfil the sanctioned posts through short-term contracted staff recruited by the regional health directorate, district health office, local governments, and non-governmental organisation. At the time of assessment, the number of SBAs, non-SBAs, ANMs and doctors surpassed the sanctioned post except for paramedics.

The overall readiness score to provide BEmOC in Taplejung (76.8\%) was much higher than figures shown in other LMICs (Bintabara et al. 2019; Kanyangarara et al. 2018; Andriantsimietry et al. 2016). In Madagascar, district hospitals had a mean score of $60.4 \%$ and basic health centres had a 44.5\% for BEmOC services in 2014 (Andriantsimietry et al. 2016). In Tanzania, the overall readiness score for BEmOC was $40.3 \%$ (Bintabara et al. 


\section{Availability of emergency obstetric care}

2019). A study that assessed the obstetric service readiness in 17 LMICs showed that the median percentage of facilities readiness to provide EmOC was 10\% (Kanyangarara et al. 2018). However, it is important to note that there were discrepancies in the tracer items used in the domains.

A few HPs were performing assisted vaginal deliveries and manual removal of placenta but PHCCs were not because of lack of cases to perform these signal functions in the last three months in the PHCCs. The possible rational for no cases in PHCCS might be due to easy access to neighbouring district hospital and tertiary hospitals. The other reasons of unavailability of a case to perform signal functions may be the non-risk taking attitude of health workers. SBA trained nurse who are authorised to perform the signal functions independently can do so only after evaluating that a patient meets the criteria and patients with serious complications would eventually be referred to hospital. This means that health workers from health posts and PHCCs tend to refer pregnant women to a higher level of health care than handling the deliveries themselves for fear of maternal and neonatal complications is justifiable. Availability of suitably trained cadre of health workers and equipment in the birthing centres but their fear of maternal and neonatal complications and case referring attitude could be the subject of future study. It would be useful to know in the future study what kind of case the HPs, and PHCCs refer to the tertiary health care system.

Although our study indicates that lack of cases as the main reason for not performing EmOC services, unavailability of essential equipment and medicine also remained vital. The study found that essential equipment and medicines for performing some EmOC functions was either missing or not functional. For example, xylocaine was not available in a place where caesarean section was carried out in district hospital as it was stocked out on the day of the 


\section{Availability of emergency obstetric care}

survey. The health personnel interviewed explained that they periodically experienced stockout of medicine and equipment needed for removal of retained products and performing assisted vaginal delivery including administration of parenteral antibiotics. The findings are consistent with evidence from studies conducted in India (Sabde et al. 2016), and Ethiopia (Ethiopian Public Health Institute et al. 2017).

Parenteral administration of anticonvulsants was the least performed signal functions which is consistent with the findings from Nepal (Ministry of Health et al. 2017), and other 17 LMICs (Kanyangarara et al. 2018). Similarly, the other signal functions least performed, i.e. removal of retained products by manual vacuum aspirations and assisted vaginal delivery (vacuum extraction) are similar to the findings of the study conducted in LMICs (MEASURE Evaluation PIMA 2016; Worku et al. 2013; Ameh et al. 2012) including Nepal (Ministry of Health et al. 2017).

\section{Limitations}

This study, which involved a survey of health facilities, observation of key items and extraction of data from health facility register, has some limitations. It is possible that staff might have been biased in providing information on the availability of the equipment, supplies and commodities to seek support and influence donor agencies working on the district. This could have affected the information on the availability, which was based on whether the equipment was available. It was to limit this response bias that we chose to observe the essential items and further categorised as observed, reported not seen and not available. To avoid information bias, the respondent of the survey was either facility incharge or responsible staff member of maternal and newborn care services. 


\section{Availability of emergency obstetric care}

The data on service utilisation was extracted from the health facility register, i.e. HMIS, held by the health facilities which is often criticised as being incomplete. It is, therefore, possible the use of the EmOC services to be exaggerated due to poor record keeping. Nevertheless, we attempted to reduce this uncertainty by liaising with the maternity ward in-charge so that she could confirm the validity of the records.

Since it was a cross-sectional study, the mere availability or unavailability of equipment, medicine and commodities at the time of survey may disguise situations when these items were generally available and were only missing at the time of the study and vice versa. In addition, we cannot report any cause and effect as the study provides only a snapshot of the availability and readiness of birthing centres in surveyed health facilities. The study does not include user behaviour as it is outside the remit of the study objective. Another limitation was that this study does not measure the quality of the services provided.

\section{Conclusions}

In Taplejung, EmOC services were below the minimum coverage level recommended by WHO and the essential items for performing some EmOC functions were either missing or not functional. There is a clear need that the Ministry of Health and Population need to upgrade the partially performing BEmOC facilities to fully functioning BEmOC services by improve supply chain of essential medicines and commodities, and emergency transport in all facilities providing delivery services. The National Health Training Centre needs to provide delivery and newborn care service guidelines to ensure that EmOC services are provided as per these national guidelines. Besides, the local level government needs to conduct subsequent periodic assessments to examine progress achieved. The future study needs to 


\section{Availability of emergency obstetric care}

focus on understanding geographic or financial barriers to care and exploring the reasons for underutilisation of EmOC services.

\section{Acknowledgements}

The authors are grateful to Taplejung District Health Office authority for granting permission to conduct this study. The authors would also like to acknowledge the nurses and health workers who gave their valuable time to the study. The authors received no funding for this study.

\section{Conflict of interest}

The authors declare that they have no conflict of interest. 


\section{References}

Ameh, C., Msuya, S., Hofman, J., Raven, J., Mathai, M., \& van den Broek, N. (2012). Status of emergency obstetric care in six developing countries five years before the MDG targets for maternal and newborn health. PLoS One, 7(12), e49938.

Anastasi, E., Borchert, M., Campbell, O. M., Sondorp, E., Kaducu, F., Hill, O., et al. (2015). Losing women along the path to safe motherhood: why is there such a gap between women's use of antenatal care and skilled birth attendance? A mixed methods study in northern Uganda. BMC Pregnancy \& Childbirth, 15(1), 287.

Andriantsimietry, S. H., Rakotomanga, R., Rakotovao, J. P., Ramiandrison, E., Razakariasy, M. E. R., Favero, R., et al. (2016). Service Availability and Readiness Assessment of Maternal, Newborn and Child Health Services at Public Health Facilities in Madagascar. African Journal of Reproductive Health, 20(3), 149-158.

Bintabara, D., Ernest, A., \& Mpondo, B. (2019). Health facility service availability and readiness to provide basic emergency obstetric and newborn care in a low-resource setting: evidence from a Tanzania National Survey. BMJ Open, 9(2), e020608.

Bohren, M. A., Hunter, E. C., Munthe-Kaas, H. M., Souza, J. P., Vogel, J. P., \& Gülmezoglu, A. M. (2014). Facilitators and barriers to facility-based delivery in low- and middleincome countries: a qualitative evidence synthesis. Reproductive Health, 11(1), 71.

Devkota, M., G. Shakya, N. Pratap K.C., M. Dariang, M. T. Upadhyay, S. Karn, et al. (2011). Readiness of Comprehensive Obstetric and Neonatal Emergency Care in Nepal. Kathmandu: National Health Sector Support Programme and Ministry of Health and Population.

Ethiopian Public Health Institute, Federal Ministry of Health, \& Averting Maternal Death and Disability. (2017). Ethiopian Emergency Obstetric and Newborn Care (EmONC) Assessment 2016. Addis Ababa, Ethiopia. 


\section{Availability of emergency obstetric care}

Family Health Division. (2002). National Safe Motherhood Plan (2002-2017). Kathmandu: Family Health Division, Government of Nepal.

Family Health Division. (2006). National Safe Motherhood and Newborn Health-Long Term Plan (2006-2017). Kathmandu: Family Health Division, Ministry of Health and Population, Government of Nepal.

Family Health Division. (2014). Results from assessing birthing centers in Nepal. Kathmandu: Family Health Division, Ministry of Health and Population, Government of Nepal.

Kanyangarara, M., Chou, V. B., Creanga, A. A., \& Walker, N. (2018). Linking household and health facility surveys to assess obstetric service availability, readiness and coverage: evidence from 17 low- and middle-income countries. Journal of Global Health, 8(1), 010603.

Karkee, R., Lee, A. H., \& Binns, C. W. (2015). Bypassing birth centres for childbirth: an analysis of data from a community-based prospective cohort study in Nepal. Health Policy and Planning, 30(1), 1-7.

Lawn, J. E., Kinney, M., Lee, A. C., Chopra, M., Donnay, F., Paul, V. K., et al. (2009). Reducing intrapartum-related deaths and disability: can the health system deliver? International Journal of Gynaecology and Obstetrics, 107 Suppl 1, S123-142.

MEASURE Evaluation PIMA. (2016). Health Facility Readiness to Provide Emergency Obstetric and Newborn Care in Kenya: Results of a 2014 Assessment of 13 Kenyan Counties with High Maternal Mortality. Nairobi, Kenya: MEASURE Evaluation PIMA, University of North Carolina at Chapel Hill.

Ministry of Health. (2016a). Annual Report, Department of Health Services 2071/2072 (2014/2015). Kathmandu: Ministry of Health, Government of Nepal. 


\section{Availability of emergency obstetric care}

Ministry of Health. (2016b). Nepal's Every Newborn Action Plan. Kathmandu: Ministry of Health, Government of Nepal.

Ministry of Health. (2017). Nepal Health Sector Strategy Implementation Plan 2016-2021.

Kathmandu: Ministry of Health, Government of Nepal.

Ministry of Health, New ERA, \& ICF. (2017). Nepal Demographic and Health Survey 2016.

Kathmandu: Ministry of Health, Nepal.

Ministry of Health, New ERA, Nepal Health Sector Support Program (NHSSP), \& ICF.

(2017). Nepal Health Facility Survey 2015. Kathmandu: Ministry of Health, Government of Nepal.

Ministry of Health and Population. (2019). Annual Report, Department of Health Services 2074/2075 (2017/2018). Kathmandu: Ministry of Health and Population, Government of Nepal.

Ministry of Health and Population, New ERA, \& ICF International Inc. (2012). Nepal Demographic and Health Survey 2011. Kathmandu: Ministry of Health and Population, Nepal

Ministry of Health and Population, New ERA, \& Macro International Inc. (2007). Nepal Demographic Health Survey 2006. Kathmandu: Ministry of Health and Population, Nepal

Mkoka, D. A., Goicolea, I., Kiwara, A., Mwangu, M., \& Hurtig, A. K. (2014). Availability of drugs and medical supplies for emergency obstetric care: experience of health facility managers in a rural District of Tanzania. BMC Pregnancy \& Childbirth, 14(1), 108.

Paxton, A., Bailey, P., \& Lobis, S. (2006). The United Nations Process Indicators for emergency obstetric care: Reflections based on a decade of experience. International Journal of Gynaecology \& Obstetrics, 95(2), 192-208. 
Pradhan, A., Aryal, R. H., Regmi, G., Ban, B., \& Govindasamy, P. (1997). Nepal Family Health Survey 1996. Kathmandu: Ministry of Health, New ERA, and Macro International Inc.

Pradhan, A., Suvedi, B. K., Barnett, S., Sharma, S. K., Puri, M., Poudel, P., et al. (2010). Nepal Maternal Mortality and Morbidity Study 2008/2009. Kathmandu: Family Health Division, Ministry of Health and Population, Government of Nepal.

Rana, T. G., Chataut, B. D., Shakya, G., Nanda, G., Pratt, A., \& Sakai, S. (2007). Strengthening emergency obstetric care in Nepal: The Women's Right to Life and Health Project (WRLHP). International Journal of Gynaecology and Obstetrics, 98(3), 271-277.

Roro, M. A., Hassen, E. M., Lemma, A. M., Gebreyesus, S. H., \& Afework, M. F. (2014). Why do women not deliver in health facilities: a qualitative study of the community perspectives in south central Ethiopia? BMC Research Notes, 7(1), 556.

Sabde, Y., Diwan, V., Randive, B., Chaturvedi, S., Sidney, K., Salazar, M., \& De Costa, A. (2016). The availability of emergency obstetric care in the context of the JSY cash transfer programme in Madhya Pradesh, India. BMC Pregnancy Childbirth, 16(1), 116.

Travis, P., Bennett, S., Haines, A., Pang, T., Bhutta, Z., Hyder, A. A., et al. (2004). Overcoming health-systems constraints to achieve the Millennium Development Goals. Lancet, 364(9437), 900-906.

van Teijlingen, E., \& Hundley, V. (2005). Pilot studies in family planning and reproductive health care. BMJ Sexual \& Reproductive Health, 31(3), 219-221.

WHO. (2015). Service Availability and Readiness Assessment (SARA): An annual monitoring system for service delivery. Reference Manual. Version 2.2. Geneva: World Health Organization. 
Availability of emergency obstetric care

WHO. (2019). Maternal mortality estimates by country. World Health Organization. Retrieved from http://apps.who.int/gho/data/view.main.1390?lang=en.

WHO, UNFPA, UNICEF, \& AMDD. (2009). Monitoring emergency obstetric care: a handbook. Geneva: World Health Organization.

Worku, A. G., Yalew, A. W., \& Afework, M. F. (2013). Availability and components of maternity services according to providers and users perspectives in North Gondar, northwest Ethiopia. Reproductive Health, 10, 43-43. 
Availability of emergency obstetric care

\section{Tables}

Table 1. Health facilities performing EmOC signal functions in three months prior to assessment.

\begin{tabular}{|c|c|c|c|c|}
\hline \multirow[b]{2}{*}{ Signal function } & \multirow{2}{*}{$\begin{array}{c}\text { All } \\
\text { facilities } \\
\%(n=16)\end{array}$} & \multicolumn{3}{|c|}{ Type of health facility } \\
\hline & & $\begin{array}{c}\text { HP \% } \\
(n=13)\end{array}$ & $\begin{array}{l}\text { PHCC \% } \\
(n=2)\end{array}$ & $\begin{array}{l}\mathrm{DH} \% \\
(\mathrm{n}=1)\end{array}$ \\
\hline \multicolumn{5}{|l|}{ BEmOC signal functions } \\
\hline $\begin{array}{l}\text { Administer parenteral } \\
\text { antibiotics }\end{array}$ & $37.5(6)$ & $23.1(3)$ & $100.0(2)$ & $100.0(1)$ \\
\hline Administer uterotonic drugs & $87.5(14)$ & $84.6(11)$ & $100.0(2)$ & $100.0(1)$ \\
\hline $\begin{array}{l}\text { Administer parentral } \\
\text { anticonvulsants }\end{array}$ & $12.5(2)$ & $0.0(0)$ & $50.0(1)$ & $100.0(1)$ \\
\hline $\begin{array}{l}\text { Perform manual removal of } \\
\text { placenta }\end{array}$ & $31.3(5)$ & $30.8(4)$ & $0.0(0)$ & $100.0(1)$ \\
\hline $\begin{array}{l}\text { Perform removal of retained } \\
\text { products }\end{array}$ & $25.0(4)$ & $15.4(2)$ & $50.0(1)$ & $100.0(1)$ \\
\hline $\begin{array}{l}\text { Perform assisted vaginal } \\
\text { delivery }\end{array}$ & $31.3(5)$ & $30.8(4)$ & $0.0(0)$ & $100.0(1)$ \\
\hline Perform newborn resuscitation & $50.0(8)$ & $46.2(6)$ & $50.0(1)$ & $100.0(1)$ \\
\hline \multicolumn{5}{|l|}{ CEmOC signal functions } \\
\hline Perform blood transfusion & $6.3(1)$ & $0.0(0)$ & $0.0(0)$ & $100.0(1)$ \\
\hline Perform caesarean section & $6.3(1)$ & $0.0(0)$ & $0.0(0)$ & $100.0(1)$ \\
\hline
\end{tabular}

EmOC: emergency obstetric care; BEmOC: basic emergency obstetric care; CEmOC:

comprehensive emergency obstetric care; HP: health post; PHCC: primary health care centre; DH: district hospital 
Table 2. Utilization of maternal and newborn health services in $\mathbf{1 6}$ health facilities in fiscal year 2016/17.

\begin{tabular}{|c|c|c|c|c|}
\hline \multirow[b]{2}{*}{ Service indicators } & \multirow{2}{*}{$\begin{array}{c}\text { All } \\
\text { facilities }\end{array}$} & \multicolumn{3}{|c|}{ Type of health facility } \\
\hline & & HP (\%) & PHCC (\%) & $\mathrm{DH}(\%)$ \\
\hline $\begin{array}{l}\text { Number of first ANC visits as per } \\
\text { protocol }\end{array}$ & 1020 & $512(50.2)$ & $105(10.3)$ & 403 (39.5) \\
\hline $\begin{array}{l}\text { Number of fourth ANC visits as per } \\
\text { protocol }\end{array}$ & 637 & $391(61.4)$ & $66(10.4)$ & $180(28.3)$ \\
\hline $\begin{array}{l}\text { Number of pregnant women who } \\
\text { received Td2 }\end{array}$ & 912 & $491(53.8)$ & $44(4.8)$ & 377 (41.3) \\
\hline $\begin{array}{l}\text { Number of new pregnant women who } \\
\text { received iron and folic acid } \\
\text { (combined tablets) }\end{array}$ & 1265 & 649 (51.3) & $86(6.8)$ & $530(41.9)$ \\
\hline $\begin{array}{l}\text { Number of women who received de- } \\
\text { worming tablets }\end{array}$ & 1228 & $610(49.7)$ & $88(7.2)$ & $530(43.2)$ \\
\hline Number of institutional deliveries & 1056 & $340(32.2)$ & $54(5.1)$ & $662(62.7)$ \\
\hline $\begin{array}{l}\text { Number of SBA delivery in facility or } \\
\text { at home by facility staff }\end{array}$ & 906 & $317(35.0)$ & $51(5.6)$ & $538(59.4)$ \\
\hline \multicolumn{5}{|l|}{ Complications seen } \\
\hline Pre-eclampsia/eclampsia & 14 & $1(7.1)$ & $0(0.0)$ & $13(92.9)$ \\
\hline Puerperal sepsis & 12 & $1(8.3)$ & $0(0.0)$ & $11(91.7)$ \\
\hline Postpartum haemorrhage & 37 & $4(10.8)$ & $1(2.7)$ & $32(86.5)$ \\
\hline Prolonged labour & 12 & $4(33.3)$ & $0(0.0)$ & $8(66.7)$ \\
\hline Retained placenta & 7 & $6(85.7)$ & $1(14.3)$ & $0(0.0)$ \\
\hline Ectopic pregnancy & 9 & $0(0.0)$ & $0(0.0)$ & $9(100.0)$ \\
\hline Other & 3 & $3(100.0)$ & $0(0.0)$ & $0(0.0)$ \\
\hline
\end{tabular}

HP: health post; PHCC: primary health care centre; DH: district hospital; ANC: antenatal care; Td: tetanus diphtheria 
Table 3. Percentage distribution of selected equipment for EmOC in 16 health facilities

\begin{tabular}{|c|c|c|c|c|}
\hline \multirow[b]{2}{*}{ Equipment } & \multirow{2}{*}{$\begin{array}{c}\text { All } \\
\text { facilities } \\
\%(n=16)\end{array}$} & \multicolumn{3}{|c|}{ Type of health facility } \\
\hline & & $\begin{array}{c}\text { HP \% } \\
(n=13)\end{array}$ & $\begin{array}{c}\text { PHCC \% } \\
(\mathrm{n}=2)\end{array}$ & $\begin{array}{l}\text { DH \% } \\
(\mathrm{n}=1)\end{array}$ \\
\hline Emergency transport & $43.8(7)$ & $38.5(5)$ & $50.0(1)$ & $100.0(1)$ \\
\hline Sterilization equipment & $93.8(15)$ & $92.3(12)$ & $100.0(2)$ & $100.0(1)$ \\
\hline Examination light & $81.3(13)$ & $76.9(10)$ & $100.0(2)$ & $100.0(1)$ \\
\hline Delivery pack & $87.5(14)$ & $84.6(11)$ & $100.0(2)$ & $100.0(1)$ \\
\hline $\begin{array}{l}\text { Suction apparatus (mucus } \\
\text { extractor) }\end{array}$ & $31.3(5)$ & $23.1(3)$ & $50.0(1)$ & $100.0(1)$ \\
\hline Manual vacuum extractor & $81.3(13)$ & $76.9(10)$ & $100.0(2)$ & $100.0(1)$ \\
\hline Vacuum aspiration or MVA kit & $87.5(14)$ & $84.6(11)$ & $100.0(2)$ & $100.0(1)$ \\
\hline Neonatal bag and mask & $81.3(13)$ & $76.9(10)$ & $100.0(2)$ & $100.0(1)$ \\
\hline Delivery bed & $100.0(16)$ & $100.0(13)$ & $100.0(2)$ & $100.0(1)$ \\
\hline Blank partographs & $87.5(14)$ & $84.6(11)$ & $100.0(2)$ & $100.0(1)$ \\
\hline Gloves & $100.0(16)$ & $100.0(13)$ & $100.0(2)$ & $100.0(1)$ \\
\hline Infant weighing scale & $100.0(16)$ & $100.0(13)$ & $100.0(2)$ & $100.0(1)$ \\
\hline Blood pressure apparatus & $100.0(16)$ & $100.0(13)$ & $100.0(2)$ & $100.0(1)$ \\
\hline $\begin{array}{l}\text { Soap and running water or } \\
\text { alcohol based hand rub }\end{array}$ & $87.5(14)$ & $84.6(11)$ & $100.0(2)$ & $100.0(1)$ \\
\hline Resuscitation table with warmer & $25.0(4)$ & $7.7(1)$ & $100.0(2)$ & $100.0(1)$ \\
\hline Oxygen supply & $18.8(3)$ & $0.0(0)$ & $100.0(2)$ & $100.0(1)$ \\
\hline Incubator & $\mathrm{n} / \mathrm{a}$ & $\mathrm{n} / \mathrm{a}$ & $\mathrm{n} / \mathrm{a}$ & $0.0(0)$ \\
\hline Anaesthesia equipment & $\mathrm{n} / \mathrm{a}$ & $\mathrm{n} / \mathrm{a}$ & $\mathrm{n} / \mathrm{a}$ & $100.0(1)$ \\
\hline Spinal needle & $\mathrm{n} / \mathrm{a}$ & $\mathrm{n} / \mathrm{a}$ & $\mathrm{n} / \mathrm{a}$ & $100.0(1)$ \\
\hline
\end{tabular}

HP: health post; PHCC: primary health care centre; DH: district hospital; MVA: manual vacuum aspiration: n/a: not applicable 
Table 4*. Percentage distribution of selected medicines and commodities for EmOC in 16 health facilities

\begin{tabular}{|c|c|c|c|c|}
\hline \multirow[b]{2}{*}{ Medicines and commodities } & \multirow{2}{*}{$\begin{array}{c}\text { All facilities } \\
\%(n=16)\end{array}$} & \multicolumn{3}{|c|}{ Type of health facility } \\
\hline & & $\begin{array}{c}\text { HP \% } \\
(n=13)\end{array}$ & $\begin{array}{c}\text { PHCC \% } \\
(n=2)\end{array}$ & $\begin{array}{l}\mathrm{DH} \% \\
(\mathrm{n}=1)\end{array}$ \\
\hline Skin disinfectant (Chlorhexidine) & $87.5(14)$ & $84.6(11)$ & $100.0(2)$ & $100.0(1)$ \\
\hline Inj Oxytocin & $87.5(14)$ & $84.6(11)$ & $100.0(2)$ & $100.0(1)$ \\
\hline Inj Ampicillin & $43.8(7)$ & $46.2(6)$ & $0.0(0)$ & $100.0(1)$ \\
\hline Inj Gentamicin & $31.3(5)$ & $23.1(3)$ & $50.0(1)$ & $100.0(1)$ \\
\hline Inj Magnesium Sulphate & $87.5(14)$ & $84.6(11)$ & $100.0(2)$ & $100.0(1)$ \\
\hline Intravenous solution with infusion set & $87.5(14)$ & $84.6(11)$ & $100.0(2)$ & $100.0(1)$ \\
\hline Inj Xylocaine & $37.5(6)$ & $38.5(5)$ & $50.0(1)$ & $0.0(0)$ \\
\hline Inj Epinephrine & $37.5(6)$ & $30.8(4)$ & $50.0(1)$ & $100.0(1)$ \\
\hline Blood supply sufficiency & $\mathrm{n} / \mathrm{a}$ & $\mathrm{n} / \mathrm{a}$ & $\mathrm{n} / \mathrm{a}$ & $0.0(0)$ \\
\hline Blood supply safety & $\mathrm{n} / \mathrm{a}$ & $\mathrm{n} / \mathrm{a}$ & $\mathrm{n} / \mathrm{a}$ & $100.0(1)$ \\
\hline Halothane (inhalation) & $\mathrm{n} / \mathrm{a}$ & $\mathrm{n} / \mathrm{a}$ & $\mathrm{n} / \mathrm{a}$ & $0.0(0)$ \\
\hline Atropine (injectable) & $\mathrm{n} / \mathrm{a}$ & $\mathrm{n} / \mathrm{a}$ & $\mathrm{n} / \mathrm{a}$ & $100.0(1)$ \\
\hline Thiopental (powder) & $\mathrm{n} / \mathrm{a}$ & $\mathrm{n} / \mathrm{a}$ & $\mathrm{n} / \mathrm{a}$ & $0.0(0)$ \\
\hline Suxamethonium bromide (powder) & $\mathrm{n} / \mathrm{a}$ & $\mathrm{n} / \mathrm{a}$ & $\mathrm{n} / \mathrm{a}$ & $0.0(0)$ \\
\hline Inj Ketamines & $\mathrm{n} / \mathrm{a}$ & $\mathrm{n} / \mathrm{a}$ & $\mathrm{n} / \mathrm{a}$ & $100.0(1)$ \\
\hline
\end{tabular}

HP: health post; PHCC: primary health care centre; DH: district hospital; Inj: injection; n/a: not applicable 
Availability of emergency obstetric care

\section{Figures}

Figure 1 top

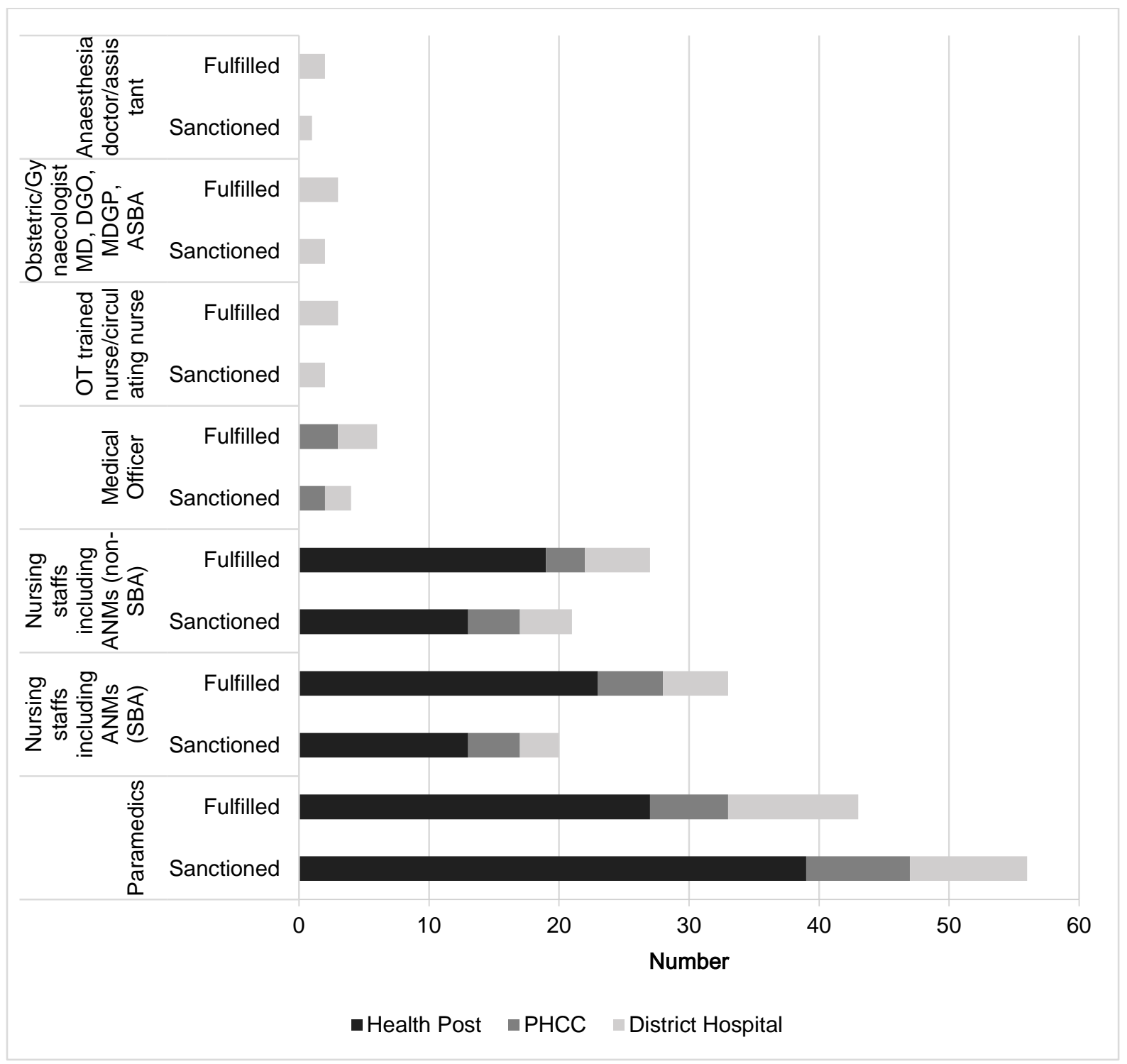


Availability of emergency obstetric care

\section{Figure 2 top}

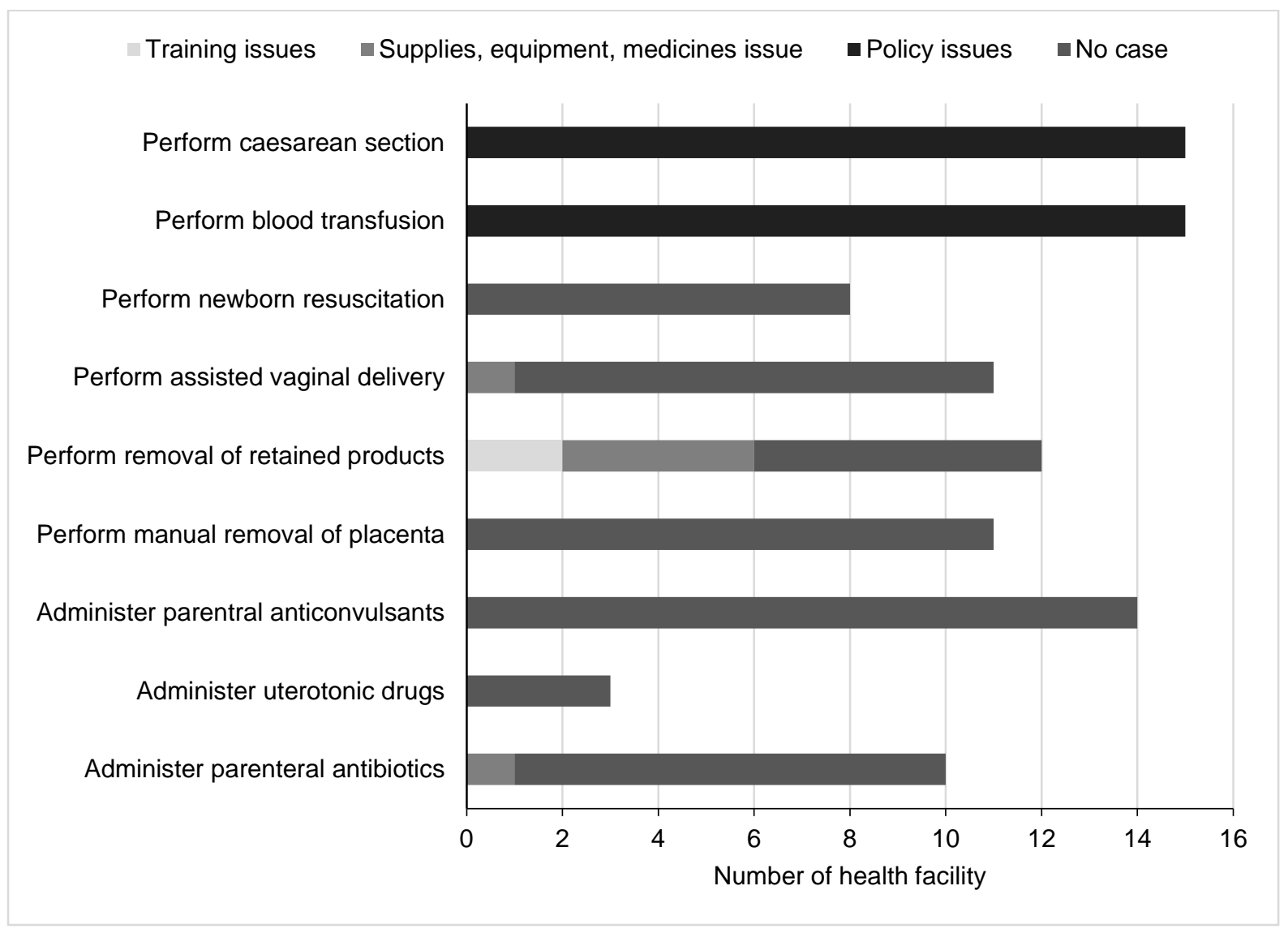




\section{Figure 3 top}

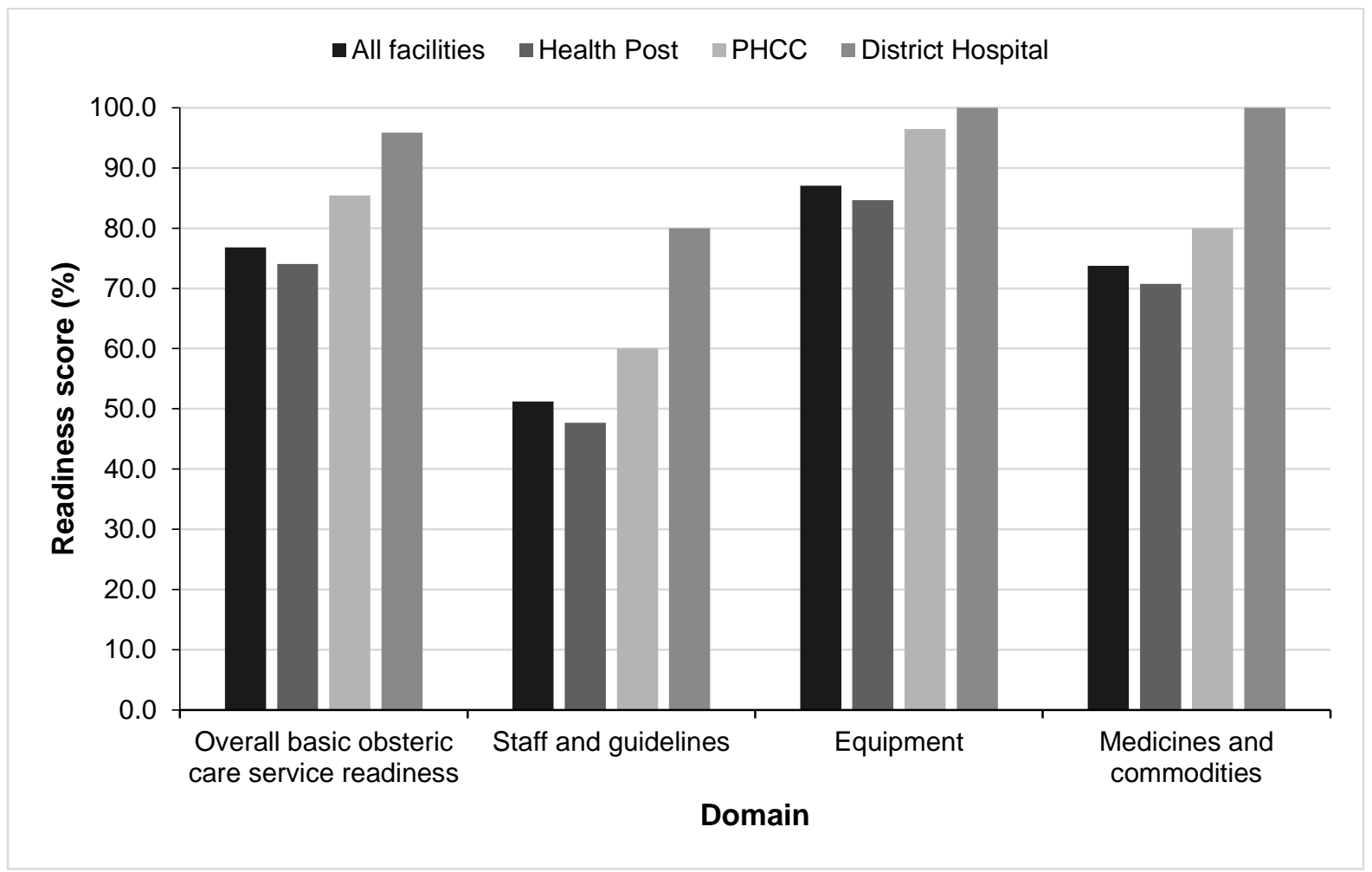

Footnotes:

Staff and guidelines included five tracer items: 1) Guidelines for essential childbirth care; 2) Checklists and/or job-aids for essential childbirth care; 3) Guidelines for essential newborn care; 4) Staff trained in essential childbirth care; and 5) Staff trained in newborn resuscitation

Equipment included 14 tracer items: 1) Emergency transport; 2) Sterilization equipment; 3) Examination light; 4) Delivery pack; 5) Suction apparatus (mucus extractor); 6) Manual vacuum extractor; 7) Vacuum aspirator or D\&C kit (with speculum); 8) Neonatal bag and mask; 9) Delivery bed; 10) Partograph; 11) Gloves; 12) Infant weighting scale; 13) Blood pressure apparatus; and 14) Soap and running water OR alcohol based hand rub Medicines and commodities included six tracer items: 1) Injectable uterotonic (oxytocin); 2) Injectable ampicillin; 3) Injectable gentamicin; 4) Magnesium sulphate (injectable); 5) Skin disinfectant (Chlorhexidine); and 6) Intravenous solution with infusion set 


\section{Figure 4 top}

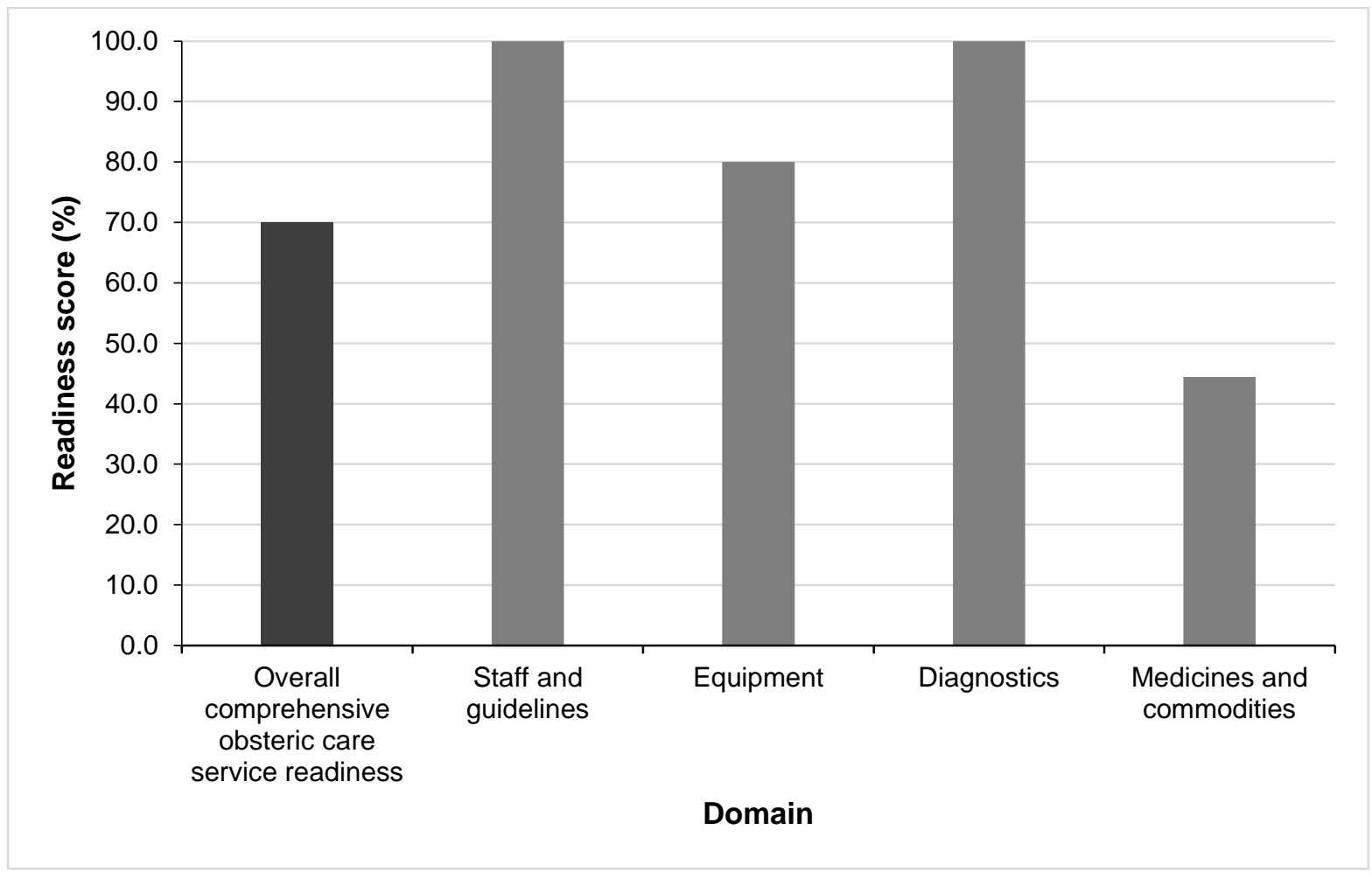

\section{Footnotes:}

Staff and guidelines included four tracer items: 1) Guidelines for CEmOC; 2) Staff trained in CEmOC; 3) Staff trained in surgery; and 4) Staff trained in anaesthesia

Equipment included five tracer items: 1) Anaesthesia equipment; 2) Resuscitation table; 3) Incubator; 4) Oxygen; and 5) Spinal needle

Diagnostics included two tracer items: 1) Blood typing; and 2) Cross match testing

Medicines and commodities included nine tracer items: 1) Blood supply sufficiency; 2) Blood supply safety; 3) Inj Xylocaine; 4) Epinephrine (injectable); 5) Halothane (inhalation); 6) Atropine (injectable); 7) Thiopental (powder); 8) Suxamethonium bromide (powder); and 9) Ketamine (injectable) 
Availability of emergency obstetric care

\section{Supplementary Figure S1 top}

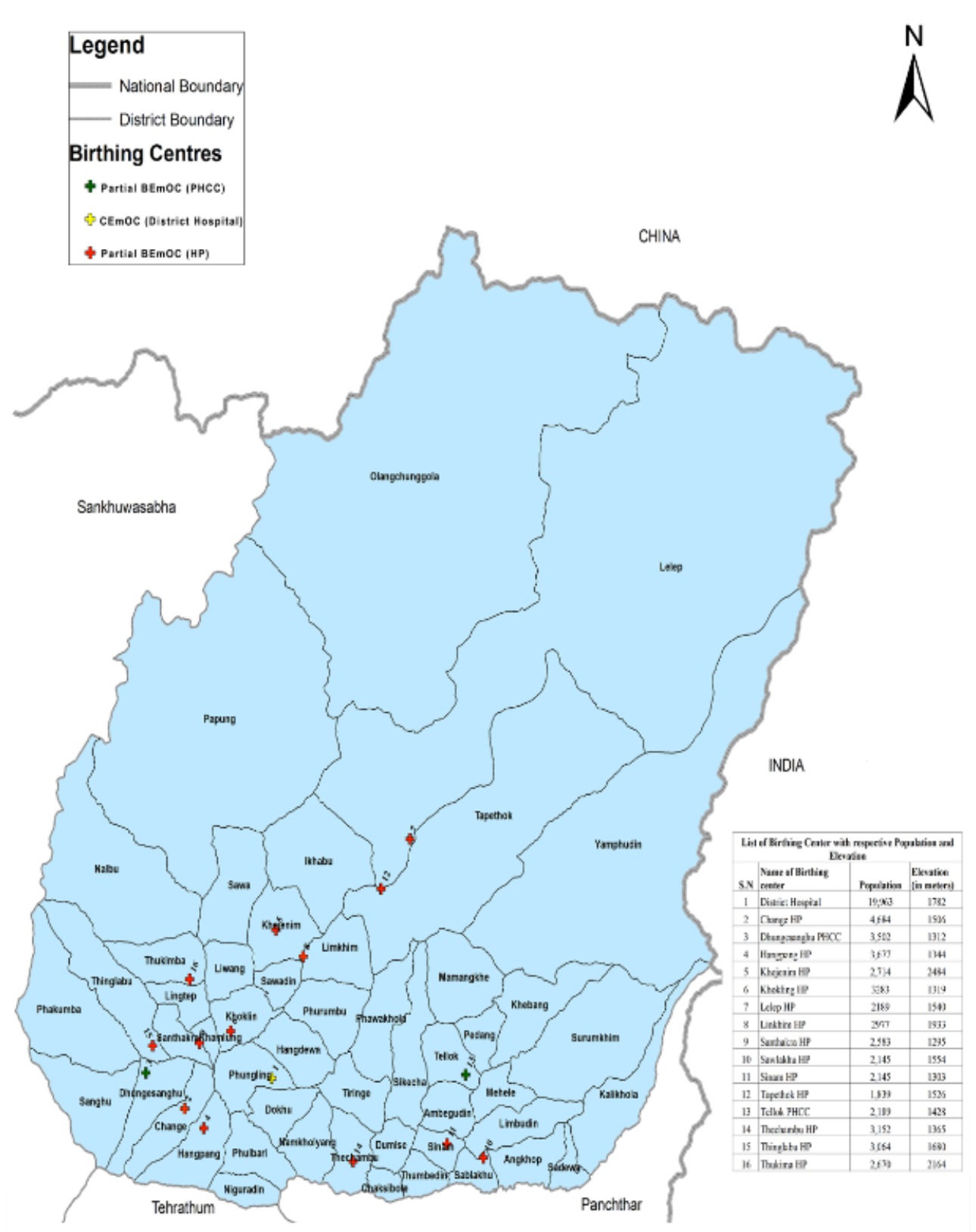


Availability of emergency obstetric care

\section{Figure Captions}

Figure 1. Number of health workers at the time of assessment in birthing centres, Taplejung 2018.

Figure 2. Reasons for not performing EmOC signal functions in three months prior to assessment, Taplejung $2018(\mathrm{n}=16)$.

Figure 3. Overall facility readiness scores for BEmOC

Figure 4. Overall facility readiness scores for CEmOC

Supplementary Figure S1. District populations and distribution of birthing centres in Taplejung, 2017. 